\title{
Qualidade de vida e o desenvolvimento de depressão e ansiedade em estudantes de medicina de uma instituição de ensino da Amazônia
}

\author{
Quality of life and the development of depression and anxiety \\ medical students of an institution of teaching of the Amazon
}

\begin{abstract}
Angélica Arêa Leão Martins', Fabíola Gonçalves Andrade', Ícaro Giovanni Sousa De Albuquerque ${ }^{1}$, Paulo Batista Da Costa Neto', Thais Sant' ana Soares Silva', Leonardo Teixeira de Mendonça'
\end{abstract}

\begin{abstract}
Resumo Objetivo: Identificar e compreender a qualidade de vida e o desenvolvimento de depressão e ansiedade em estudantes de uma Instituição de Ensino da Amazônia (IES) no município de Belém, estado do Pará, no ano de 2017. Método: Foi realizado estudo observacional, individual, descritivo e transversal, com alunos de uma IES da Amazônia, através dos questionários WHOQOL e escala HAD, com uso dos testes de Mann Whitney e TStudent para análises comparativas, sendo os demais dados analisados por estatística simples. Resultados: Dos 126 estudantes avaliados, houve predomínio de jovens acima de 19 anos, do sexo feminino, sem graduações anteriores. Observou-se diferença estatisticamente significante na avaliação do questionário WHOQOL nos domínios de meio ambiente e social, sendo em ambos os resultados do $1^{\circ}$ semestre pior que o $7^{\circ}$ semestre; Também foi significativo o resultado dos sintomas possíveis de transtornos de ansiedade, sendo esses sintomas mais prevalentes nos alunos do $1^{\circ}$ semestre (63\% vs $48 \%$ ). Os demais domínios do WHOQOL e resultados do HAD não apresentaram diferenças, mas se observou alta prevalência de sintomas de depressão tanto no $1^{\circ}$ quanto no $7^{\circ}$ semestre ( 49 e $35 \%$ respectivamente). Conclusão: Os dados encontrados estão em consonância com a literatura, e mostram que mesmo com melhoras em alguns quesitos relacionados a qualidade de vida no decorrer da graduação, a presença de elevados índices de triagem para transtornos do humor (depressão e ansiedade) dentre esse grupo populacional merece atenção e mais estudos para mudar essa realidade e o futuro desses profissionais.

Descritores: qualidade de vida; depressão; ansiedade; estudantes de medicina.
\end{abstract}

Summary Purpose: To identify and understand the quality of life and the development of depression and anxiety in students of an Amazonian Teaching Institution (IES) in the city of Belém, state of Pará, in the year 2017. Methods: An individual observational study, descriptive and cross-sectional study, with students from an IES of the Amazon, using the WHOQOL and HAD-scale questionnaires, using the Mann Whitney and T-Student tests for comparative analysis, and the other data were analyzed by simple statistics. Results: Of the 126 students evaluated, there was a predominance of girls over 19 years of age, female, without previous graduations. A statistically significant difference was observed in the evaluation of the WHOQOL questionnaire in the environmental and social domains, with both results of the 1st semester worse than the 7th semester; It was also significant the result of the possible symptoms of anxiety disorders, being these symptoms more prevalent in the first semester students (63\% vs $48 \%$ ). The other domains of the WHOQOL and HAD results showed no differences, but there was a high prevalence of symptoms of depression in both the 1st and the 7th semester (49 and 35\%, respectively). Conclusion: The data found are in line with the literature, and show that even with improvements in some aspects related to quality of life during graduation, the presence of high screening indices for mood disorders (depression and anxiety) among this group population deserves attention and more studies to change this reality and the future of these professionals.

Keywords: quality of life; depression; anxiety; medical students.

${ }^{1}$ Faculdade Metropolitana da Amazônia - FAMAZ, Belém, PA, Brasil

Fonte de financiamento: Estudo autofinanciado.

Conflito de interesses: Os autores declararam não haver conflitos de interesse.

Recebido: Abril 15, 2018

Aceito: Maio 28, 2018

Trabalho realizado na Faculdade Metropolitana da Amazônia - FAMAZ, Belém, PA, Brasil.

Copyright Martins et al. Este é um artigo publicado em acesso aberto (Open Access) sob a licença Creative Commons Attribution, que permite uso, distribuição e reprodução em qualquer meio, sem restrições desde que o trabalho original seja corretamente citado. 


\section{Introdução}

O termo qualidade de vida vem sofrendo alterações com o passar do tempo. Desde a década de 30 até a de 90 no século passado, foi definido como "[...] a percepção do indivíduo de sua posição na vida, no contexto da cultura e sistema de valores nos quais ele vive e em relação aos seus objetivos, expectativas, padrões e preocupações [...]"1. Considerando a graduação em medicina, observa-se a extensa procura por êxito profissional e pessoal, o qual, em muitos casos, é convertido em frustações pelo não alcance almejado e a baixa qualidade de vida. Contribui para tal, também, o fenômeno da "idealização do curso", na qual a graduação é apenas objeto de resultados benéficos na esfera social, financeira, acadêmica e profissional, desprezando o trajeto laboroso, inerente ao curso $^{2}$. Nesse sentido, esse contexto propicia o desenvolvimento de alterações do humor (depressão e ansiedade) nesse grupo.

A OMS define Ansiedade como "[...] um sentimento vago e desagradável de medo, apreensão, caracterizado por tensão ou desconforto derivado de antecipação de perigo, de algo desconhecido ou estranho [... $]^{\prime \prime 3}$. Segundo o DSM-V é uma "antecipação da ameaça futura. Além disso, a Depressão é caracterizada como "[...] transtorno mental comum, caracterizado por tristeza, perda de interesse, ausência de prazer, oscilações entre sentimentos de culpa e baixa autoestima, além de distúrbios do sono ou do apetite. Também há a sensação de cansaço e falta de concentração [... $]^{\prime \prime 3-6}$.

Ambos distúrbios têm sido observados em estudantes e posteriormente em médicos em prevalências cada vez maiores. Um dos gatilhos iniciais para o desenvolvimento desses transtornos começa no pouco conhecimento acerca da rotina na graduação e/ou profissional, impactando de um modo negativo no dia-a-dia e qualidade de vida do estudante ao se deparar com as realidades complexas do curso ${ }^{7}$.

Nesse sentido, a qualidade de vida possui fatores controlados pelos indivíduos - administração do tempo nos estudos, descanso, lazer - ou não controlados - conteúdo acadêmico, condições de infraestrutura, segurança - com consequências no seu desenvolvimento psicossocial; as quais podem resultar em transtornos físicos e mentais, influenciando na quantidade e qualidade do sono, alimentação e relações sociais, principalmente no âmbito familiar ${ }^{8,9}$. Além disso, o ingresso no meio universitário proporciona novas relações sociais e adoção de novos comportamentos, tornando os indivíduos vulneráveis às condutas de risco à saúde, como o etilismo e o isolamento social, por exemplo ${ }^{10}$.

Desse modo, buscou-se identificar e compreender a qualidade de vida e o desenvolvimento de depressão e ansiedade em estudantes de Medicina de Instituição de Ensino da Amazônia (IES) no município de Belém, estado do Pará.

\section{Método}

Realizou-se um estudo epidemiológico de caráter observacional, individual, descritivo e transversal, em alunos da IES sendo eles do primeiro e sétimo períodos do curso de medicina, no mês de novembro de 2017, com preenchimento por 126 estudantes em sala de aula, com esclarecimentos sobre a pesquisa e assinaturas do TCLE - Termo de consentimento Livre e esclarecido.

Para a avaliação da qualidade de vida foi utilizado o questionário WHOQOL-ABREVIADO em versão português, já validada, composto por 26 perguntas de autoanalise das ultimas duas semanas, divida por domínios - físico, psicológico, relações sociais e relações ambientais -, com facetas correspondentes. Para avaliação de alterações do humor foi utilizado o questionário HAD, o qual possui 14 questões intercaladas de ansiedade e depressão, com escore de: improvável, possível e provável. Em virtude de o número ser adequado, o mesmo resultará na própria amostra, por isso não necessitou de seleção de amostra.

Os critérios utilizados para a inclusão dos sujeitos foram: ser estudante de Medicina da IES, responder o questionário. Como critérios de exclusão: ausência no dia da aplicação e o não preenchimento do termo de consentimento livre e esclarecido. Além disso, as coletas de dados foram feitas pelos pesquisadores e aconteceram após aprovação pelo CEP - comitê de Ética e Pesquisa. Sendo os resultados descritos e analisados por meio de tabelas, quadros e gráficos, analisados à luz da literatura revisada.

A análise estatística foi feita através dos softwares Bioestat 5.3 e Microsoft Excel 2010. Para análise dos dados do questionário WHOQOL com resposta baseada em escala Likert de 5 pontos, foram utilizados os testes de Mann Whitney e T-Student. Para os resultados descritivos foram calculados médias e desvios padrões, para cada quesito. Na análise da correlação entre os resultados da WHOQOL e da escala HAD, foram feitas comparações entre os domínios da WHOQOL e o resultado da escala HAD. 


\section{Resultados}

Foram abordados alunos de duas turmas, sendo apenas os formulários de 125 acadêmicos considerados, com 77 (61,6\%) alunos do primeiro semestre - ciclo básico - e 48 (38,4\%) alunos do sétimo semestre - ciclo clínico. Assim, na avaliação social, observou-se predomínio do sexo feminino (66\%), estado civil solteiro (93\%), idade acima de 19 anos (62\%), sendo padrão em ambos os períodos. A maioria (75\%) não possuía graduações anteriores, com proporção um pouco menor, ao avaliar o $7^{\circ}$ período isoladamente (Tabela 1).

$\mathrm{Na}$ análise do WHOQOL-BREF (Tabela 2), evidenciou-se diferenças estatisticamente significativas $(\mathrm{p}<0,05)$ entre o primeiro e o sétimo do semestre, relacionadas a aspectos Sociais $(\mathrm{p}=0.0007)$ e Ambientais $(\mathrm{p}=0.0441)$. Sendo nos quesitos do meio ambiente, $76 \%$ dos alunos do $1^{\circ}$ semestre e $52 \%$ dos alunos do $7^{\circ}$ assinalaram respostas negativas - em relação a segurança física e proteção, ambiente no lar, recursos financeiros, cuidados de saúde e sociais, ambiente físico e transporte. No entanto, no "domínio de relações sociais", houve apenas $34 \%$ de respostas negativas entre os alunos do $7^{\circ}$ período (alunos do $1^{\circ}$ período com $71 \%$ ) - em relações pessoais, suporte (apoio) social e atividade sexual. Não havendo diferença significante nos demais domínios nem no resultado geral.

Houve diferenças estatisticamente significativas $(\mathrm{p}=0,04)$ no domínio de Ansiedade, no qual os alunos do $1^{\circ}$ período resultaram em cerca de $63 \%$ como "possível" e "provável" de Transtorno de Ansiedade (TA), sendo os alunos do $7^{\circ}$ período com aproximadamente $48 \%$ (Tabela 3 e 4 ).

$\mathrm{Na}$ investigação de ansiedade, aproximadamente $49 \%$ dos alunos do $1^{\circ}$ semestre autoanalisaram-se "tenso ou contraído" em "boa parte" ou na "maior parte do tempo", sendo no $7^{\circ}$ semestre com cerca de $32 \%$.

Tabela 1. Avaliação Social-Demográfica

\begin{tabular}{|c|c|c|c|c|}
\hline & \multirow[t]{2}{*}{ Variáveis } & \multicolumn{2}{|c|}{ Período do curso } & \multirow{2}{*}{$\begin{array}{c}\text { Total } \\
125\end{array}$} \\
\hline & & $1^{\circ}-(\mathrm{N}=77)$ & $7^{\circ}-(\mathrm{N}=48)$ & \\
\hline \multirow[t]{2}{*}{ Sexo } & Homem & $26(33,77 \%)$ & $20(41,66 \%)$ & $46(36,8 \%)$ \\
\hline & Mulher & $51(66,23 \%)$ & $28(58,34 \%)$ & $79(63,2 \%)$ \\
\hline \multirow[t]{3}{*}{ Idade } & Acima de 19 anos & $38(49 \%)$ & $39(81 \%)$ & $77(62 \%)$ \\
\hline & Abaixo ou igual 19 anos & $30(39 \%)$ & - & $30(24 \%)$ \\
\hline & Não informaram & $9(12 \%)$ & $9(19 \%)$ & $18(14 \%)$ \\
\hline \multirow[t]{3}{*}{ Estado Civil } & Solteiro & $73(95 \%)$ & $43(90 \%)$ & $116(93 \%)$ \\
\hline & Casado & $4(5 \%)$ & $4(8 \%)$ & $8(6 \%)$ \\
\hline & Divorciado/Viúvo/União Estável & 0 & $1(2 \%)$ & $1(1 \%)$ \\
\hline \multirow[t]{3}{*}{ Graduações Anteriores } & Sim & $8(10 \%)$ & $11(23 \%)$ & $19(15 \%)$ \\
\hline & Não & $66(86 \%)$ & $28(58 \%)$ & $94(75 \%)$ \\
\hline & Não informaram & $3(4 \%)$ & $9(19 \%)$ & $12(10 \%)$ \\
\hline
\end{tabular}

Fonte: Autoria própria, dados do estudo (2018).

Tabela 2. Estatísticas referentes ao WHOQOL

\section{Características}

\begin{tabular}{lccc}
\hline \multicolumn{1}{c}{ Características } & & Período do curso \\
& $1^{\circ}-\mathrm{n}=77$ & $2^{\circ}-\mathrm{n}=48$ & $\mathrm{p}$-valor (Mann-Whitney) \\
\hline Domínio Social Mediana & 3,33 & 4 & $\mathrm{p}=0.0007$ \\
\hline Meio Ambiente Mediana & 3,5 & 3,88 & $\mathrm{P}=0.0083$ \\
\hline Psicológico Mediana & 3,5 & 3,67 & $\mathrm{p}=0.0758$ \\
\hline Percepção e Satisfação & & 4 & $\mathrm{p}=0.3085$ \\
\hline Mediana & 4 & 3 & $\mathrm{p}=0.1798$ \\
\hline Físico Mediana & 3,14 & 4 & $\mathrm{p}=0.2102$ \\
\hline Geral Mediana & 4 & & \\
\hline
\end{tabular}

Fonte: Autoria própria (2017). 
Tabela 3. Escores de avaliação da Ansiedade pelo HAD

\begin{tabular}{cccc}
\hline & & Ansiedade & \\
Período do curso & Improvável & Possível & Provável \\
\cline { 2 - 4 } & $30(39 \%)$ & $25(32 \%)$ & $22(29 \%)$ \\
\hline $1^{\circ}$ Período & $25(52 \%)$ & $16(33 \%)$ & $7(15 \%)$ \\
\hline $7^{\circ}$ Período & & Depressão & Provável \\
\hline Período do curso & Improvável & Possível & $7(9 \%)$ \\
\hline $1^{\circ}$ Período & $39(51 \%)$ & $31(40 \%)$ & $5(10 \%)$ \\
\hline $7^{\circ}$ Período & $31(65 \%)$ & $12(25 \%)$ & \\
\hline
\end{tabular}

Fonte: Autoria própria (2017).

Tabela 4. Estatísticas referentes ao HAD

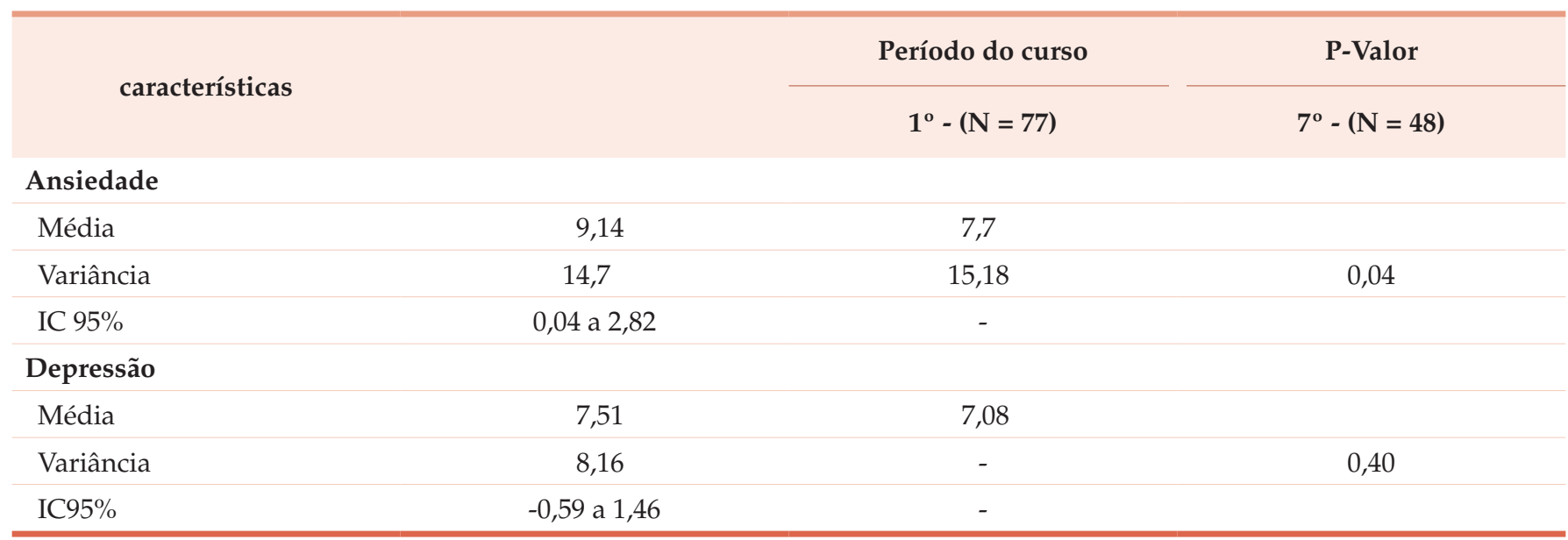

Fonte: Autoria própria (2017).

Além disso, 38\% dos alunos do $1^{\circ}$ semestre alegaram dificuldade de "sentar-se e se sentir relaxado", sendo o $7^{\circ}$ semestre com $25 \%$. Na abordagem sobe "sensação de pânico", este índice chegou a 44,15\% no $1^{\circ}$ semestre e $27,08 \%$ no $7^{\circ}$ semestre. Os resultados do HAD relacionado à "Depressão" mostraram que $49 \%$ (38) e $35 \%$ (17) dos alunos no $7^{\circ}$ e do $1^{\circ}$ período, respectivamente, possuíram escore de "possíveis" e "prováveis", não havendo diferença significativa na comparação entre os períodos.

Foram realizadas análises multivariadas correlacionando o gênero, idade, e dados sociais (estado civil, graduações anteriores), todavia nenhuma correlação obteve resultados estatisticamente significantes.

\section{Discussão}

É notório que a qualidade de vida é alicerçada em numerosos quesitos, como a saúde física e psicológica, a independência, os relacionamentos sociais e, também, no meio ambiente ${ }^{11}$. Neste contexto, obtiveram-se divergências em comparação com a pesquisa realizada em Recife com alunos de medicina, a qual se pontuou decréscimo relevante no domínio "psicológico" em alunos na conclusão do curso, enquanto que na presente pesquisa houve aumento da média do domínio nos alunos do $7^{\circ}$ período em relação aos do $1^{\circ}$ período ${ }^{12}$. Desse modo, infere-se que fatores como localidade regional e tempo de experiência e adaptação no curso são importantes; e, holisticamente, os distúrbios psiquiátricos são decorrentes de uma gama de fatores, dentre eles a idade - geralmente são jovens em maturação psicológica - a exigência pela "manutenção" do sucesso e o manejo da profissão - de contato com situações clinicas traumáticas ${ }^{7,8,13}$.

Na análise sobre o "suporte e ao apoio social", o sétimo semestre apresentou uma avaliação mais positiva, assim como em "Atividade Sexual". Nesse sentido, em conformidade a tais dados, o estudo 
realizado no Encontro Cientifico de Estudantes de Medicina, evidencia que o fator "relações sociais" possui tendência positiva de avaliação de satisfação no decorrer do curso ${ }^{2}$. Além disso, alunos com dificuldade em relacionamentos possuem 11 vezes mais chances de manifestar depressão comparada àqueles com "bom" controle relacionamento ${ }^{14}$.

Houve significância em relação à "atividade de recreação e lazer", o qual o primeiro semestre apresentou maior insatisfação, a qual pode ser reflexo do maior nível de exigência, tanto do próprio aluno quanto da faculdade - na supervalorização de conteúdos e o desempenho acadêmico em um curto período de tempo - em detrimento da manutenção da qualidade de vida9. Assim, afetando com insegurança, cansaço e tristeza, refletindo no tempo destinado a família e amigos, e exercícios de corpo e mente ${ }^{2,9,15}$. Além da exigência de profissionais comprometidos e com autocontrole, em muitos casos, menosprezando sua saúde mental ${ }^{16}$.

Houve maior satisfação - maior escore - das "condições do local de moradia", pelos alunos do sétimo período; seguindo também, nesse sentido, o acesso aos serviços de saúde. Estando, assim, em consonância com a pesquisa com estudantes de medicina de Brasília, dos quais 70,2\% estavam satisfeitos/muito satisfeitos com sua saúde; e outro realizado com estudantes paulistas, avaliando em $74 \%$ positivamente a qualidade da saúde 9 . Tal questão é de suma importância, haja vista que, por exemplo, no quesito de violência, o DATASUS alega aumento da mortalidade no Pará em acidentes de transito, sendo $6.850 / 2015^{17}$, o que pode influenciar na preocupação com a integridade física e moral individual.

Observou-se maior presença de sintomas de transtorno da ansiedade nos alunos do $1^{\circ}$ semestre, avaliados pela escala HAD. Pôde-se inferir que o ingresso na universidade pode gerar instabilidade, resultando em preocupação, dúvida e ansiedade em relação ao processo de ensinoaprendizagem ${ }^{18}$. Os dados obtidos da pesquisa diferem da literatura, os quais afirmam o aumento do nível de ansiedade com o decorrer do curso, principalmente entre alunos de medicina e da área da saúde ${ }^{19,20}$. Revelando o quão alto são os índices de prevalência, talvez pela alta cobrança individual e institucional, demonstrando a necessidade de maior atenção e análise pelas instituições de ensino superior.

Vale ressaltar a elevação dos sinais e sintomas de ansiedade no estudo e o possível desenvolvimento de transtorno comportamental, principalmente no primeiro semestre, revelam que a saúde mental ainda converge menos atenção, e que a prevenção e a sua manutenção são "relegadas", influenciando de modo negativo na integridade pessoal e no desempenho acadêmico. Além disso, o resultado do domínio de depressão foi preocupante haja vista que a mesma pode cursar com ideações e tentativas - falhas ou com "sucesso" - de suicídio. Cerca de 800 mil pessoas morrem por suicídio a cada ano - sendo a segunda principal causa de morte na faixa etária de 15 a 29 anos $^{21}$.

No que tange a períodos de adaptação, a Ansiedade pode ser transitória, sendo, principalmente, em momentos de mudanças abruptas, como no início da vida acadêmica e no internato. Nesse contexto, foram observadas exposições constantes a situações estressantes, como o medo e os desafios do mercado de trabalho, os quais aumentam a suscetibilidade a surtos emocionais. Vale ressaltar que $15 \%$ a $25 \%$ dos estudantes de medicina evidenciaram algum sofrimento psiquiátrico ${ }^{22-24}$. Além disso, Depressão e Ansiedade predispõem a relações conturbadas, uso de drogas, diminuição do desempenho acadêmico e da empatia, além de ideação suicida ${ }^{18,25}$.

\section{Conclusões}

Desse modo, os dados encontrados estão em consonância com a literatura, e mostram que mesmo com melhoras em alguns quesitos relacionados a qualidade de vida no decorrer da graduação, a presença de elevados índices de triagem para transtornos do humor (depressão e ansiedade) dentre esse grupo populacional merece atenção e mais estudos para mudar essa realidade e o futuro desses profissionais, através de melhores politicas internas institucionais e auxilio aos jovens em formação.

\section{Agradecimentos}

Agradecemos: A Deus, o qual nos ajudou em todos os aspectos, do trabalho e da vida; A cada estudante de medicina participante e que contribuiu para o projeto. 


\section{Referências}

1. Fleck MPA. The World Health Organization instrument to evaluate quality of life (WHOQOL-100): characteristics and perspectives. Ciênc. saúde coletiva vol.5 no.1 Rio de Janeiro 2000. http://dx.doi.org/10.1590/S1413-81232000000100004.

2. Fiedler PT. Avaliação da qualidade de vida do estudante de medicina e da influência exercida pela formação acadêmica [tese]. São Paulo (SP): Faculdade de Medicina; 2008. http://dx.doi.org/10.11606/T.5.2008.tde-10072008-161825.

3. Castillo ARGL, Recondo R, Asbahr FR, Manfro GG. Transtornos de ansiedade. Rev Bras Psiquiatr. 2000;22(Supl 2):20-3.

4. American Psychiatric Association. Transtornos de ansiedade. In: American Psychiatric Association. DSM-5: manual diagnóstico e estatístico de transtornos mentais. 5. ed. Porto Alegre: Artmed; 2014. p. 189-234.

5. Fundação Oswaldo Cruz. Destaques: mais de 350 milhões de pessoas sofrem de depressão no mundo [Internet]. Rio de Janeiro: Fiocruz; 2018 [citado em 2018 maio 20]. Disponível em: http://www.canal.fiocruz.br/destaque/index.php?id=722

6. Abelha L. Depressão, uma questão de saúde pública. Cad Saude Colet. 2014;22(3):223-223. http://dx.doi.org/10.1590/1414-462X201400030001.

7. Feodrippe ALO, Brandao MCF, Valente TCO. Qualidade de vida de estudantes de Medicina: uma revisão. Rev Bras Educ Med. 2013;37(3):41828. http://dx.doi.org/10.1590/S0100-55022013000300014.

8. Vasconcelos TC, Dias BRT, Andrade LR, Melo GF, Barbosa L, Souza E. Prevalência de sintomas de ansiedade e depressão em estudantes de Medicina. Rev Bras Educ Med. 2015;39(1):135-42. http://dx.doi.org/10.1590/1981-52712015v39n1e00042014.

9. Bampi LNS, Baraldi S, Guilhem D, Araújo MP, Campos ACO. Qualidade de vida de estudantes de medicina da Universidade de Brasília. Rev Bras Educ Med. 2013;37(2):217-25. http://dx.doi.org/10.1590/S0100-55022013000200009.

10. Queiroz Brito BJ, Gordia AP, Quadros TMB. Revisão da literatura sobre o estilo de vida de estudantes universitários. Rev Bras Qual de Vida. 2014;6(2):66-76.

11. World Health Organization Quality of Life. The development of the World Health Organization quality of life assessment instrument (the WHOQOL). Quality of life assessment: international perspectives. Geneva: WHO; 1994. p. 41-57.

12. Alves JGB, Tenório M, Anjos AG, Figueroa JN. Qualidade de vida em estudantes de Medicina no início e final do curso: avaliação pelo Whoqol-bref. Rev Bras Educ Med. 2010;34(1):91-6. http://dx.doi.org/10.1590/S0100-55022010000100011.

13. Marques-Deak A, Sternberg E. Psiconeuroimunologia: a relação entre o sistema nervoso central e o sistema imunológico. Rev Bras Psiquiatr. 2004;26(3):143-4. http://dx.doi.org/10.1590/S1516-44462004000300002. PMid:15645058.

14. Zonta R, Robles ACC, Grosseman S. Estratégias de enfrentamento do estresse desenvolvidas por estudantes de Medicina da Universidade Federal de Santa Catarina. Rev Bras Educ Med. 2006;30(3):147-53. http://dx.doi.org/10.1590/S0100-55022006000300005.

15. Alves AAM, Rodrigues NFR. Determinantes sociais e económicos da Saúde Mental. Rev Port Saude Publica. 2010;28(2):127-31. http:// dx.doi.org/10.1016/S0870-9025(10)70003-1.

16. Paula JA, Borges AMFS, Bezerra LRA, Parente HV, Paula RCA, Wajnsztejn R, et al. Prevalência e fatores associados à depressão em estudantes de medicina. Rev Bras Crescimento Desenvolv Hum. 2014;24(3):274-81.

17. Departamento de Informática do SUS. Óbitos por causas externas [Internet]. Rio de Janeiro: DATASUS; 2018 [citado em 2018 mar 24 ]. Disponível em: http://tabnet.datasus.gov.br/cgi/tabcgi.exe?sim/cnv/ext10uf.def/

18. Cardozo MQ, Gomes KM, Fan LG, Soratto MT. Fatores associados à ocorrência de ansiedade dos acadêmicos de Biomedicina. Rev Saúde e Pesquisa. 2016;9(2):251-62. http://dx.doi.org/10.17765/1983-1870.2016v9n2p251-262.

19. Abrão CB, Coelho EP, Passos LBS. Prevalência de sintomas depressivos entre estudantes de medicina da Universidade Federal de Uberlândia. Rev Bras Educ Med. 2008;32(3):315-23. http://dx.doi.org/10.1590/S0100-55022008000300006.

20. Alves TCTF. Depressão e ansiedade entre estudantes da área de saúde. Rev Med. 2014;93(3):101-5.

21. Organização Pan-Americana da Saúde. Organização Mundial da Saúde. Aumenta o número de pessoas com depressão no mundo [Internet]. Brasília: OPAS/OMS; 2017 [citado em 2018 mar 24]. Disponível em: http://www.paho.org/bra/index.php?option=com_content\&view=art icle\&id=5354:aumenta-onumero-de-pessoas-com-depressao-no-mundo\&ltemid=839

22. Tabalipa FO, Souza MF, Pfützenreuter G, Lima VC, Traebert E, Traebert J. Prevalence of anxiety and depression among medical students. Rev Bras Educ Med. 2015;39(3):388-94. http://dx.doi.org/10.1590/1981-52712015v39n3e02662014.

23. Gonçalves A, Freitas P, Sequeira C. Comportamentos suicidários em estudantes do ensino superior: factores de risco e de protecção. Millenium. 2011;40:149-59.

24. Wander DCO. O sofrimento psíquico relacionado ao trabalho do enfermeiro em ambiente hospitalar [monografia]. Santa Cruz do Sul (RS): Universidade de Santa Cruz do Sul; 2016.

25. Enns MW, Cox BJ, Sareen J, Freeman P. Adaptive and maladaptive perfectionism in medical students: a longitudinal investigation. Med Educ. 2001;35(11):1034-42. http://dx.doi.org/10.1046/j.1365-2923.2001.01044.x. PMid:11703639. 


\section{Autor correspondente}

Fabíola Gonçalves Andrade

Av. Pedro Miranda, 1712, casa 22

CEP 66080-500, Belém, PA, Brasil

Tel.: (91) 99160-1506

E-mail: fabiolaandrade-23@hotmail.com

\section{Informação sobre os autores}

AALM, FGA, IGSA, PBCN e TSSS são graduandos do curso de Bacharelado em Medicina pela Faculdade Metropolitana da Amazônia (FAMAZ).

LTM é especialista com Residência médica em Clinica médica pelo Hospital Municipal Dr. Carmino Caricchio (HMCC); especialista com Residência médica em Reumatologia na Universidade Federal de São Paulo (UNIFESP); Mestrado Profisional em Reumatologia pela Universidade Federal de São Paulo (UNIFESP); docente em Habilidades Clínicas e em Reumatologia Geral, da Faculdade Metropolitana da Amazônia (FAMAZ).

\section{Contribuição dos autores}

AALM, FGA e ÍGSA participaram e efetuaram todas as etapas da elaboração da pesquisa e confeç̧ão do manuscrito; PBCN e TSSS participaram e efetuaram todas as etapas da elaboração da pesquisa e confecção do manuscrito; LTM participou na concepção inicial, acompanhamento, orientação do estudo e dos resultados encontrados, além da elaboração e revisão crítica do manuscrito.

Todos os autores leram e aprovaram a versão final submetida ao Pará Research Medical Journal. 